\title{
A Group Key Management Scheme Based on Random Transmission for VANET
}

\author{
Liang PANG ${ }^{1, b}$, Da WEI ${ }^{1,2}$, Qi ZHAO ${ }^{3}$, Jianqi ZHU ${ }^{1,2, a}$ \\ ${ }^{1}$ College of Computer Science and Technology, Jilin University, Changchun, 130012, China \\ ${ }^{2}$ Key Laboratory of Symbolic Computation and Knowledge Engineering of Ministry of Education, \\ Jilin University, Changchun, 130012, China \\ ${ }^{3}$ Jilin University, Changchun, 130012, China \\ aemail: zhujq@jlu.edu.cn, bemail:13654408367@163.com
}

Keywords: VANET Group; Random Transmission; Polynomial; Hash Chain

\begin{abstract}
Group key management plays an important role in ensuring the safety and reliability of the VANET (vehicular ad-hoc network) over the channel. Through considering the characteristics of VANET communication and topology, a VANET group key management scheme is proposed based on random transmission which is combined with the construction of polynomial and key updating by Hash chain. The scheme achieves the random transmission function of group key and the node revocation capability. Moreover, it can ensure the forward and backward and other security attributes of the group key. The results show that the proposed scheme has less communication complexity and better communication performance, also it can suitable for large scale VANET group.
\end{abstract}

\section{Introduction}

Secure group communication has been an active research domain in recent years. Since most group communication between vehicles takes place over wide-open expanse of Internet, to encrypt data is a major consideration. Therefore, a group key management scheme needs to be proposed.

The first group key agreement was proposed by Steer et al. in 1988[1]. With the improvement of network technology, the group key management is divided into three basic types: centralized group key management, distributed group key management and decentralized group key management. The centralized group key management has a central organization used for the generation and distribution of key. Guo et al. presented a centralized group key management mechanism for VANET [2]. The distributed group key management divides the group into several sub groups. And each group has a coordinator to manage its own group. Wu et al. proposed a distributed key management based on cipher broadcast [3]. There is no central body in the decentralized group key management and member nodes have the equal status. Guo et al. presented a decentralized group key management by creating hierarchical key tree [4].

However, in VANET environment high mobility of vehicles will result in frequent changes of network topology. And high mobility or stagnation at any moment of vehicles will effect network link based on location to be randomness. Thus typical distributed group key management or centralized group key management can’t be applied to large scale VANET group communication.

In this paper, we proposed a group key management scheme based on random transmission for VANET. The scheme based on random transmission has the following characteristics: (1) Only communication with nearby nodes when sharing data between groups; (2) We can use non group member nodes to transfer message; (3) The message transfer path is adaptive stochastic; (4) Compared with the centralized key management mechanism, the single point failure problem can be avoided effectively; (5) Compared to the typical distributed key agreement mechanism, the number of communication in VANET can be effectively reduced.

We organize the rest paper as follows. In Section 2, we give out group key management scheme. In section 3, we discuss the efficiency of our key agreement. Finally, we conclude the paper. 


\section{Group Key Management Scheme Based on Random Transmission}

In this section, we present detailed method of group key management scheme for VANET. The scheme mainly includes five aspects: system initialization, group key exchange, group key generation, group key distribution and group key update. The overall process is shown in Figure 1.

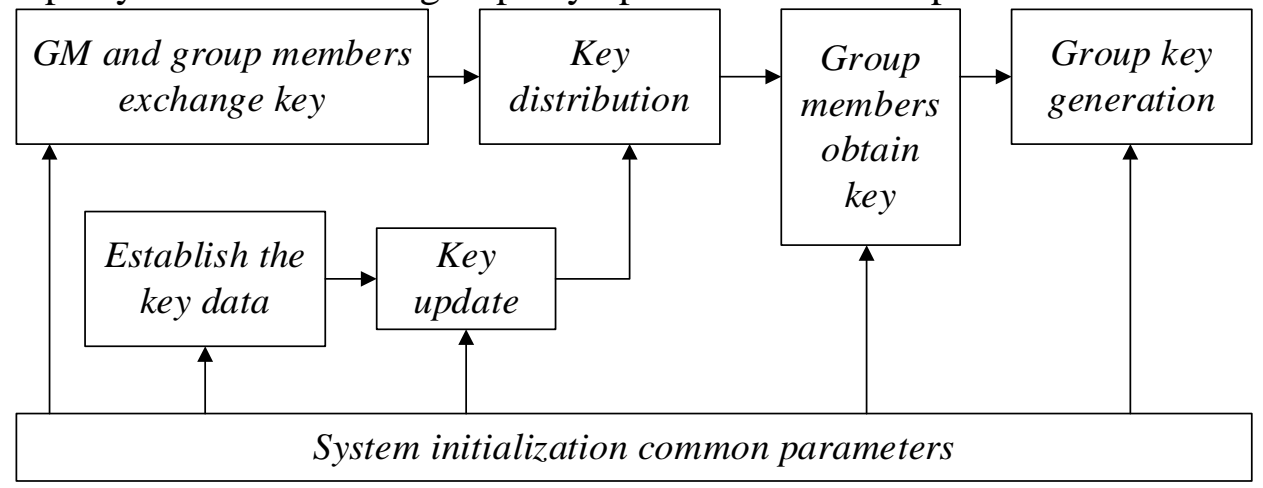

Fig 1. Process of group key management scheme

\subsection{System Initialization}

The symbol definition is given out of the scheme shown in Table 1 . Vehicle node $V_{i}$ accesses network, registers and applies for $\operatorname{Cert}\left(V_{i}\right)$ from $C A$ (Certificate Authority). After the application has been approved by $C A$, the $\operatorname{Cert}\left(V_{i}\right)=\left\{\operatorname{Sig}_{C A}\left(P K_{V_{i}} \| I D_{V_{i}}\right), P K_{V_{i}}, I D_{V_{i}}, P K_{C A}\right\}$ will be sent to $V_{i}$. $\operatorname{Sig}_{C A}\left(P K_{A} \| I D_{A}\right)$ represents digital signature from $C A$ to data. After more than one vehicle access network, $V_{i} \ldots V_{n}$ compose Group $_{s}=\left\{G M_{s}, V_{i} \ldots V_{n} \mid i, n, s \in N\right\} . V_{i}$ communicates with $G M_{s}$ by RSU (Road Side Unit). $G M_{s}$ selects nodes to participate into the collection $U_{\text {neg }}=\left\{V_{\text {neg }} \mid V_{\text {neg }} \in\right.$ Group $\left._{s}\right\}$ according to nodes' online time and $L_{i i}$. $L_{i i}$ represents recent assessment value of OBU (On board Unit). $G M_{s}$ verifies $\operatorname{Cert}\left(V_{i}\right)$ and agrees prime finite fields $F_{q}$ with $V_{i} . F^{+}{ }_{q}$ is cyclic group of qth-order addition, the bit length of $q$ is $b=\left(\log _{2} q+1\right) / 2, g$ is $F^{+}$'s generator. Message authentication code algorithms are defined as $M A C_{A}\left(\bullet^{\bullet}\right)$ and $M_{A} C_{B}\left(\bullet^{\bullet}\right)$. Export key function is $K F(x)=H(X \| j), j$ represents counter.

Table 1. Symbol definition of scheme

\begin{tabular}{|c|c|c|c|}
\hline Symbol & Symbol definition & Symbol & Symbol definition \\
\hline Group $_{s}$ & Vehicle Group S & $M_{T}$ & Key message \\
\hline$V_{i}$ & Vehicle Node $V_{i}$ & List $_{\text {Add }}^{T-1, T}$ & Member increment list \\
\hline $\operatorname{Cert}\left(V_{i}\right)$ & $V_{i}$ certificate & ${ }_{\text {ist }}^{\text {Aad }} T-1, T$ & Мेe \\
\hline$G M_{s}$ & Group Manager & $\operatorname{LISt}_{\text {sub }}$ & Member decrement list \\
\hline$s_{G M_{s}}$ & $G M_{s}$ private key & $L_{\text {List }}$ & Key chain length \\
\hline$P_{G M_{s}}$ & $G M_{s}$ public key & $K_{L}$ & Hash chain \\
\hline$S_{V_{i}}$ & $V_{i}$ private key & $\operatorname{Key}_{S}^{T_{\text {seq }}}$ & $T_{\text {seq }}$ group key \\
\hline$P_{V_{i}}$ & $V_{i}$ public key & $T_{K}^{\min }$ & Minimum undate \\
\hline$k_{s}^{i}$ & Key factor & $V_{d e l}$ & 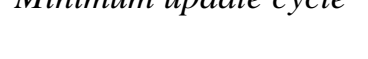 \\
\hline$K_{s}^{T}$ & Key data & dele & Node to be deleted \\
\hline$I D_{V}^{i}$ & $V_{i}$ Node identifier & $\boldsymbol{1}_{K}$ & Force update cycle \\
\hline List $_{S}^{T}$ & $T$ cycle members & $B_{S}^{I_{\text {seq }}}$ & Apply for broadcast \\
\hline
\end{tabular}

\subsection{Group Key Exchange}

In this section, group key exchange is based on the mBPR model [5]. Assuming that the private and public key of $G M_{s}$ are $\left(s_{G M_{S}}, P_{G M_{S}} \mid P_{G M_{S}}=g^{s_{G M_{s}}} \bmod q\right)$, the private and public key of $V_{i}$ are $\left(s_{V_{i}}, P_{V_{i}} \mid P_{V_{i}}=g^{s_{V_{i}}} \bmod q\right)$. In this formula, $\operatorname{gcd}\left(s_{G M}, q-1\right)=1$ and $\operatorname{gcd}\left(s_{V_{i}}, q-1\right)=1$. The process 
of group key exchange between $G M_{s}$ and $V_{i}$ is as follows:

(1) $V_{i}$ selects a random $a_{i} \in Z_{q}^{*}$ and sets $r_{i}=P_{V_{i}}^{a_{i}} \bmod q . V_{i}$ selects $k_{i} \in Z_{q}^{*}$ and computes $s_{i}=g^{-k_{i}} r_{i}^{s_{V_{i}}} \bmod q$ and $t_{i}=\left(1-s_{V_{i}}\right) a_{i}+s_{V_{i}}{ }^{-1}\left(k_{i}-s_{i}\right) \bmod (q-1)$, sends $r_{i}, s_{i}, t_{i}$ to $G M_{s}$;

(2) $G M_{s}$ executes the same process as (1) and sends $r_{G M}, s_{G M}, t_{G M}$ to $V_{i}$;

(3) $G M_{s}$ receives $r_{i}, s_{i}, t_{i}$ data and computes $\left(P_{V_{i}}\right)^{t_{i}}=r_{i}^{\left(1-s_{V_{i}}\right)} g^{\left(k_{i}-s_{i}\right)} \bmod q$. If $\left(P_{V_{i}}\right)^{t_{i}} s_{i} g^{s_{i}}=r_{i}$, computes $k_{S}^{i}=\left(r_{i}\right)^{s_{G M} a_{G M}} \bmod q$. Otherwise, taking a renegotiation;

(4) $\mathrm{Vi}$ executes same process as (3), and computes $k_{S}^{G M}=\left(r_{G M}\right)^{s_{i} a_{i}} \bmod q$. The key factor $k_{S}^{i}=k_{S}^{G M}=g^{a_{i} a_{G M} S_{V_{i}} S_{G M}}$ is shared among all the vehicles in the group.

\subsection{Group Key Generation}

Group key generation is based on the mKY model [5]. $V_{i}$ selects a random $x_{i}$ as private key, so the public key is $Y_{i}=g^{x_{i}} \bmod q$. The process of setting up the current group key is as follows:

(1) $V_{i}$ selects a random $a_{i} \in Z_{q}^{*}$, computes $s_{i}=a_{i} g^{x_{i}} \bmod q-\left(r_{i}+g^{x_{i}} \bmod q\right) x_{i} \bmod (q-1)$ and $r_{i}=g^{a_{i}} \bmod q . V_{i}$ broadcasts $M_{i}=\left(\right.$ seq, $r_{i}, s_{i}$, Cert $\left._{i}\right)$ to other $U_{\text {neg }}$ members;

(2) At the same time, $V_{i}$ receives $M e s=\left\{M_{1}, \ldots, M_{i-1}, M_{i+1}, \ldots, M_{n}\right\}$ from other members. For example, $V_{i}$ receives $V_{j}$ 's data $M_{j}$ and computes $Y_{j}^{\prime}=\left(g^{x_{j}} \bmod q\right)^{r_{j}+g^{x_{j}} \bmod q} g^{s_{j}} \bmod q$. If $Y_{j}^{\prime}=r_{i}^{Y_{j}}$, set $\quad g_{i}=r_{i+1} / r_{i-1} . V_{i}$ computes $s_{i}^{\prime}=x_{i}-\left(g_{i}^{a_{i}} \bmod q+g^{x_{i}} \bmod q\right)\left(x_{i}+a_{i}\right) \bmod (q-1) \quad$ and $Z_{i}=g_{i}^{a_{i}} \bmod q$, broadcasts $M_{i}^{\prime}=\left(\right.$ seq, $Z_{i}, s_{i}^{\prime}$, Cert $\left._{i}\right)$. If $Y_{j}^{\prime} \neq r_{i}^{Y_{j}}$, end computational process;

(3) $V_{i}$ receives $M e s^{\prime}=\left\{M_{1}^{\prime}, \ldots, M_{i-1}^{\prime}, M_{i+1}^{\prime}, \ldots, M_{n}^{\prime}\right\}$ from second round and computes $Y_{j}^{\prime \prime}=\left(r_{j} Y_{j}\right)^{Z_{j}+Y_{j}}(g)^{s_{j}^{\prime}} \bmod q$. If $Y_{j}^{\prime \prime}=Y_{j}, V_{i}$ computes $Y_{j}^{\prime \prime}=r_{i}^{n a_{i}} Z_{i}^{n-1} Z_{i+1}^{n-2} \cdots Z_{i+n-2}^{0} \bmod q$ to get the key. Otherwise, end computational process.

\subsection{Key Distribution Based on Random Transmission}

Collection List $_{T}=\left\{V_{i}, V_{i} \in\right.$ Group $\left._{s}\right\}$ represents the nodes which are to be distributed in circle $T$. We use $K_{S}^{T}$ to distribute the key data based on constructing polynomial.

(1) $G M_{s}$ queries the collection $K_{s}^{F}=\left\{k_{s}^{1}, k_{s}^{2}, \cdots k_{s}^{m}, m \in N^{+}, 5 \leq m\right\}$ and constructs the polynomial in finite field $F_{q}: F_{q}^{1}(x)=\left(\left(x-k_{s}^{1}\right)\left(x-k_{s}^{2}\right) \cdots\left(x-k_{s}^{m}\right)+K_{s}^{T}\right) \bmod q$.Expanding the polynomial based on congruence of number theory:

$F_{q}^{1}(x)=\left(x^{m} \bmod q+a_{1} x^{m-1} \bmod q+\cdots+a_{m-1} k_{s}^{1} k_{s}^{2}+\cdots+k_{s}^{m} \bmod q+K_{s}^{T} \bmod q\right) \bmod q$.

In this formula, $a_{1}, a_{2} \cdots, a_{m-1}$ is according to the specific key factor parameters to calculate. $G M_{s}$ computes $K_{*}^{1}=a_{m-1} k_{S}^{1} k_{S}^{2} \cdots k_{s}^{m} \bmod q+K_{s}^{T} \bmod q$ and expands polynomial $F_{q}^{1}(x)$ to $F_{q}^{1}(x)=\left(x^{m} \bmod q+a_{1} x^{m-1} \bmod q+\cdots+K_{*}^{1}\right) \bmod q . G M_{s}$ broadcasts $M_{T}=\left\{a_{1}\left|a_{2}\right| \cdots\left|K_{*}^{1}\right| q \mid m\right\}$;

(2) $\mathrm{Vi}$ in the ist $_{S}^{T}$ receives $M_{T}$ and constructs the polynomial by the same coefficient: $F_{q}^{\prime}(x)=\left(x^{m} \bmod q+a_{1} x^{m-1} \bmod q+\cdots+K_{*}^{1}\right) \bmod q$.

$V_{i}$ sets $k_{S}^{i}$ as $x$ value into $F_{q}^{\prime}(x)$ and records as $K_{T}=F_{q}^{\prime}\left(k_{S}^{i}\right)$. Now $V_{i}$ gets key data $K_{S}^{T}$ from Group $_{s}$. The process of key distribution based on constructing polynomial shown in Figure 2. 


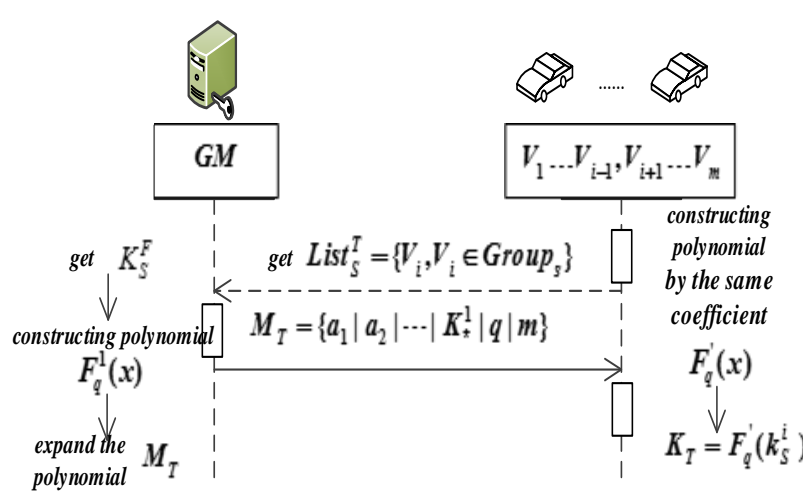

Fig 2. Process of key distribution

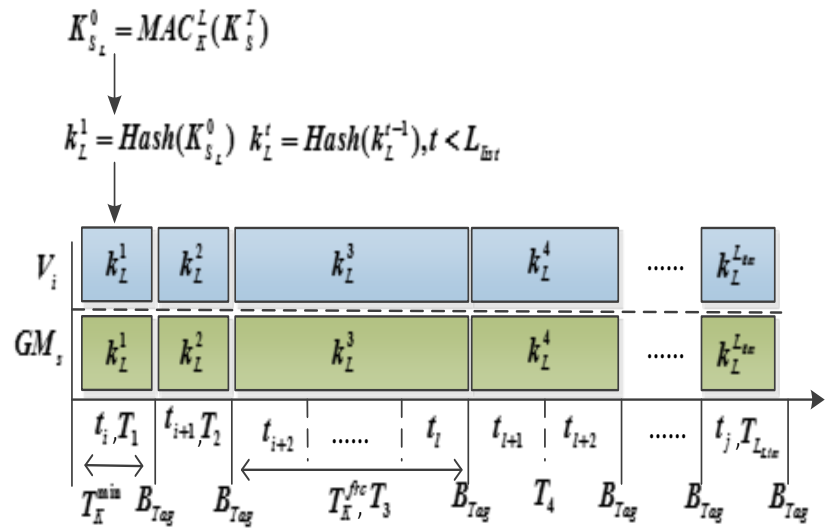

Fig 3.Process of key data update

The nodes got $K_{T}$ are defined as $U_{c h v}=\left\{V_{c h v}^{i} \mid V_{c h v}^{i} \in \operatorname{Group}_{s}\right\}$, conversely the collection is $U_{\text {unch }}=\left\{V_{\text {unch }}^{i} \mid V_{\text {unch }}^{i} \in\right.$ Group $\left._{s}\right\}$. The process of transmission is as follows:

(1) $V_{\text {unch }}^{i}$ does not obtain $K_{s}^{T}$, then monitors broadcast data nearby itself. If $V_{\text {unch }}^{i}$ does not obtain $K_{s}^{T}$ after monitoring for a while, it will randomly connect with other vehicles in the $U_{c h v}$ which are close in physical space to apply for the key data;

(2) The vehicle in the $U_{c h v}$ receives the application. If the vehicle has already get the data $M_{T}$, it will transfer $M_{T}$ to $V_{\text {unch }}^{i}$. $V_{\text {unch }}^{i}$ gets $M_{T}$ and analyzes out key data $K_{s}^{T}$ by method mentioned in section 2.4.1. Now $V_{u n c h}^{i}$ gets $K_{s}^{T}$ and it will be added to $U_{c h v}$. Then $V_{c h v}^{i}$ storages $M_{T}$;

(3) If other vehicle $V_{\text {unch }}^{j}$ applies for $M_{T}$ from $V_{c h v}^{i}, V_{c h v}^{i}$ will transfer $M_{T}$ to $V_{\text {unch }}^{j}$.

\subsection{Group Key Update}

The process of key data update based on Hash chain shown in Figure 3.

(1) $G M_{s}$ selects Hash function $\mathrm{H}_{\mathrm{K}}^{\mathrm{L}}(\bullet)$ which is resisted strong collision, selects message authentication code function $M A C_{K}^{L}$ and digital signature algorithm $\operatorname{Sig}(\bullet)$, computes $L_{L i s t}=T_{K}^{\text {frc }} / T_{K}^{\min }$ as the length of key. $G M_{s}$ constructs $B_{\text {para }}=\left\{\mathrm{H}_{\mathrm{K}}^{\mathrm{L}}\left\|T_{K}^{\min }\right\| T_{K}^{\text {frc }}\|\operatorname{Sig}\| L_{L i s t}\right\}$ and broadcasts $B_{\text {para }}$ to all the group members;

(2) $G M_{s}$ divides periodic time series $T_{K}^{i, j}=\left\{t_{i}, t_{i+1}, \cdots, t_{j} \mid i, j \in N^{+}\right\}$and computes $K_{S_{L}}^{0}=M A C_{K}^{L}\left(K_{S}^{T}\right)$, and then distributes $K_{S_{L}}^{0}$ to group members. $G M_{s}$ and $V_{i}$ compute $K_{L}=\left\{k_{L}^{1}, k_{L}^{2}, \cdots, k_{L}^{L_{\text {list }}}\right\}$ with $K_{S_{L}}^{0}$ by using $\mathrm{H}_{\mathrm{K}}^{\mathrm{L}}(\bullet)$ function, while $t<L_{\text {List }}, k_{L}^{t}=\operatorname{Hash}\left(k_{L}^{t-1}\right)$;

(3) When group members change or $T_{K}^{\min }$ is reaches, $G M_{s}$ triggers key update event and updates $B_{\text {Tag }}=\left\{\right.$ Group $_{s}|\operatorname{tag}| T_{\text {seq }} \mid \operatorname{Sig}_{s_{G M}}\left(\right.$ Group $\left.\left._{s}|\operatorname{tag}| T_{\text {seq }}\right)\right\}, T_{\text {seq }}$ is current time period;

(4) $V_{i}$ receives $k_{s}^{T_{s e q}}$ in the next circle of $K_{L}$, uses key derivation function $K F(x)$ to compute $\mathrm{Key}_{S}^{T_{\text {seq }}}$ in circle $T_{\text {seq }}: \operatorname{Key}_{S}^{T_{\text {seq }}}=K F\left(k_{L}^{T_{\text {seq }}}\right)$.

When vehicle nodes join in the group, $G M_{s}$ distributes $k_{L}^{T_{\text {seq+1 }}}$ which is in circle $T_{\text {seq+1 }}$ to $G_{\text {new }}^{S}$ and broadcasts $B_{\text {Tag }}$. The new joined node $V_{j}$ receives $k_{L}^{T_{\text {seq+1 }}}$ and constructs incomplete Hash chain $K_{L}^{T_{\text {seq }}}$, computes $\mathrm{Key}_{S}^{T_{\text {seq+1 }}}$ in circle $T_{\text {seq+1 }}$ by using formula 2.3. $V_{i}$ receives $B_{\text {Tag }}$ and computes $\mathrm{Key}_{S}^{T_{\text {seq+1 }}}$ in circle $T_{\text {seq+1 }}$ by using formula (1.1).

When vehicle nodes leave the group, $G M_{S}$ sets the new collection $\operatorname{Group}_{S}^{T_{\text {seq+1 }}}$ in circle $T_{\text {seq+1 }}$. $G M_{s}$ computes $K_{L}^{t}=M A C_{K}^{L}\left(k_{L}^{T_{\text {seq }}}\right)$ and distributes $K_{L}^{t}$ as new cipher key to $\operatorname{Group}_{S}^{T_{\text {seq }+1}} \cdot V_{j}$ in $\operatorname{Group}_{S}^{T_{\text {seq+1 }}}$ gets $k_{s}^{T_{\text {seq }}+1}$ and computes $\mathrm{Key}_{S}^{T_{\text {seq+1 }}}$ by using formula (1.1). 


\section{Scheme Safety and Performance Analysis}

\subsection{Safety Analysis}

In this scheme, group key exchange and generation has already been proved in security based on mBPR [6] model and mKY [6] security model. The node has not exchanged key factor with $G M_{s}$ can't compute key data. Any node $V_{i}$ can compute $k_{s}^{i}$ with a non-ignorable probability $\varepsilon$, it also can solve high order polynomial problem of Ruffini Abel theorem or large number factorization problem with a non-ignorable probability. So the security of group key is guaranteed.

The described key updating mechanism in this scheme is based on Hash chain. Hash has the features of unidirectional and resistance to strong collision. Any node has got key data in circle $T_{\text {seq }}$ wants to get the key data in its last circle $T_{\text {seq- } 1}$ is infeasible. In other words, the process of computing a last node from a current node in Hash chain is not feasible in the calculation.

When vehicle nodes leave group in circle $T_{\text {seq }}, G M_{s}$ uses the private key to computes message authentication code and tags the key data as abolished. Any node left the group can't get $K_{L}^{t}$ by updating. While $G M_{s}$ redistributes key data based on constructing polynomial, there is no key factor of the node left the group, so it can't recover the key data from the broadcast. Therefore, our scheme can effectively satisfied the forward and backward of the group key in this scheme.

Table 2. Overhead of establishing group key

\begin{tabular}{|c|c|c|c|}
\hline \multirow{2}{*}{ Scheme } & \multicolumn{3}{|c|}{ Index } \\
\hline & Communication & Calculation & Storage \\
\hline \multirow{2}{*}{ LKH } & \multirow{2}{*}{$n(U)+B$} & \multirow{2}{*}{$\cdots \cdots$} & $G M: 2 n-1$ \\
\hline & & & $V_{i}: \log _{2}(n)+1$ \\
\hline TR-GKA & $6 n(U)$ & $\begin{array}{c}\left(3 M U L+\left(n^{2}+3 n\right) / 2-3\right) M U L \\
2 s i g+(2 n-2) v s i g\end{array}$ & $2 n$ \\
\hline \multirow{2}{*}{ LMA } & $\mathrm{m}(B)$ & $G M: \mathrm{m}+2 k(M U L)$ & $G M: 3$ \\
\hline & $(2 k+1) B$ & $V_{i}: 1(M U L)$ & $V_{i}: 2$ \\
\hline \multirow{2}{*}{$\begin{array}{l}\text { Random } \\
\text { transmission }\end{array}$} & $G M: B+n(U)$, neg $: m$ & $G M:\left(n^{2}-n\right) / 2(M U L)+\operatorname{sig}$ & $G M: n$ \\
\hline & $V_{i}:$ random $\leq n$ & $V_{i}:(6+n)(E X P)+n(M U L)$ & $V_{i}: 1$ \\
\hline
\end{tabular}

Table 3. Overhead of key update by joining notes into the group

\begin{tabular}{ccc}
\hline \multirow{2}{*}{ Scheme } & \multicolumn{2}{c}{ Index } \\
\cline { 2 - 3 } LKH & $2 \log _{2} n(M), 1(U)$ & Calculation \\
\hline TR-GKA & $6 n(U)$ & $\left(3 M U L+\left(n^{2}+3 n\right) / 2-3\right) M U L$ \\
& & $2 s i g+(2 n-2) v s i g$ \\
LMA & $1(S B), 1(U), 2(B)$ & $G M: k(M U L)$ \\
Random & $G M: B$ & $V_{i}: 1($ MUL $)$ \\
transmission & $V_{i}:$ random $\leq n$ & $G M: 1($ HASH $)$ \\
\hline \hline
\end{tabular}

Table 4. Overhead of key update by revoking notes out of the group

\begin{tabular}{l} 
Scheme \\
\cline { 2 - 3 }
\end{tabular}

LKH $\quad 2 \log _{2} n(M)$

\begin{tabular}{ccc} 
TR-GKA & $6 n(U)$ & $\left(3 M U L+\left(n^{2}+3 n\right) / 2-3\right) M U L$ \\
LMA & $1(S B), 2(B)$ & $2 s i g+(2 n-2) v s i g$ \\
Random & $\begin{array}{c}G M: B+n, \\
n e g: m\end{array}$ & \\
transmission & $V_{i}:$ random $\leq n$ & $V_{i}:(6+n)(E X P)+n(M U L)$ \\
\hline \hline
\end{tabular}

\subsection{Performance Analysis}

In this section, we compare LKH [6] agreement base on logic key tree, TR-GKA [5] agreement and LMA [7] scheme which is similar in structure with this paper. We take the overall number of communication rounds and the calculation of a single node as the main reference.

Assuming that $n$ is the number of group member nodes, $m$ is the number of nodes participated in group key data negotiation. MUL represents point multiplication of elliptic curve, $B$ is global 
broadcast, $U$ is unicast communication of point to point, EXP is modular exponentiation, Hash represents one-way Hash operation, MAC is message authentication code operation, sig is digital signature, vsig is validate of digital signature, random is random communication process. The overhead of establishing group key in communication, calculation and storage shown in Table 2.

From table 2, it is clear that our scheme has larger calculation than LMA agreement, GM needs polynomial times multiplication, one time digital signature. $V_{i}$ has the same scale in calculation with TR-GKA agreement needs modular exponential operation. In storage aspect, our scheme is better than LKH agreement and TR-GKA agreement, and at the same level with LMA agreement. It has the same scale with other schemes in communication performance.

When there are vehicle nodes joined or left the group, the key update mode is different from key establishment phase. Overhead of key update by joining notes into the group or revoking notes out of the group shown in Table 3 and 4. From table 3 and 4, our scheme has better performance than other schemes in communication. And when nodes join into the group, our scheme is superior to others in calculation. But when nodes left the group, members that did not leave the group using the method of redistribution in our scheme. So it is more complex than other schemes.

\section{Conclusion}

In this paper, our scheme based on random transmission is better reflected in safety and communication performance. The key path is depth random of this scheme. When the number of group members increases linearly, the frequency of the communication will grow exponentially in distributed group key management. But communication times still appeared linear growth in our scheme. And propagation delay time is logarithmic growth. Under ideal circumstances, the number of nodes in a single cycle will be exponential growth in this paper. That means group key can quickly cover the key data and be applied to large scale VANET communication requirements.

\section{Acknowledgement}

In this paper, the research was sponsored by the National Natural Science Fund (Project No. 61373123 and 61572229).

\section{References}

[1] D. Steer, L. Strawczynski, W. Diffie, M. Wiener. “A Secure Audio Teleconference System,” Advances in Cryptology (CRYPTO’88), 1988:520-528

[2] Guo M H, Liaw H T, Deng D J, et al. Centralized group key management mechanism for VANET[J].Security and Communication Networks,2013, 6(8):1035-1043.

[3] Wu Q, Qin B, Zhang L, et al. Fast transmission to remote cooperative groups: a new key management paradigm[J].Networking, IEEE/ACM Transactions on,2013,21(2):621-633.

[4] Ming-Huang Guo, Horng-Twu Liaw, Meng-Yu Chiu, et al. On decentralized group key management mechanism for vehicular ad hoc, networks [J]. Security \& Communication Networks, 2016, 9(3):241-247.

[5] Zhang Hua, Wen Qiao-yan, Jin Zheng-ping. Provable Safety Theory and Protocol [M]. Beijing: Science Press, 2012.304-320.

[6] Wallner D, Harder E, Agee R. Key management for multicast: Issues and architectures[R].RFC 2627, 1999.

[7] Cao Shuai, Zhang Chuan-rong, Song Cheng-yuan. Group key management scheme for large mobile Ad hoc network [J]. Application Research of Computers, 2012, 29(4):1420-1423. 